\title{
Limitations of molecularly targeted therapy
}

\author{
Corresponding author: \\ Anna Bajek, Chair of Urology, \\ Department of Tissue Engineering, \\ Collegium Medicum, Nicolaus \\ Copernicus University, Karlowicza 24, \\ 85-092 Bydgoszcz, Poland, \\ e-mail: a bajek@wp.pl
}

Medical Research Journal 2019;

Volume 4, Number 2, 99-105

10.5603/MRJ.a2019.0016

Copyright @ 2019 Via Medica

ISSN 2451-2591

\begin{abstract}
Personalized medicine is an extension of traditional medicine is based on a highly individual approach to each patient. One of the most important tools that allow this approach is targeted therapy. It focuses mainly on blocking cancer cell's proliferation and angiogenesis capabilities by interfacing with specific molecules that are involved in the growth and progression of the tumour. Small-molecule inhibitors and monoclonal antibodies are the main drugs that are currently in use in order to affect the specific biochemical pathways in cancer cells. However, likewise any other cancer therapies, targeted therapy has its own limitations. For instance, identifying a molecular target needed to begin treatment is one of those hardships. A specific molecule is crucial in this way of treatment. The other limitation is the toxicity that appears during the treatment, the same as in the case of traditional chemotherapy and radiotherapy. Furthermore, the cost of this therapy is significantly higher compared to classical treatments. However, the main obstacles are mechanisms of cancer drug resistance which are often developing in response to given drugs. In many cases, it makes further treatment impossible. This article is focusing on the limitations of molecularly targeted therapy.

Key words: molecularly targeted therapy, anti-cancer drugs, cancer drug resistance, the toxicity of anti-cancer drugs
\end{abstract}

Med Res J 2019; 4 (2): 99-105

\section{Introduction}

The purpose of modern anticancer therapies is to overcome the difficulties resulting from insufficient knowledge of the tumour genome, its high diversity and instability. Rapidly expanding knowledge of molecular biology and cancer genetics has provided us with tools that make personalized medicine possible. Molecularly targeted therapy focuses on the identification of molecular changes on the level of a single patient, and also on optimization and individualization of treatment, as well as individual characteristics of the tumour microenvironment [1-3].

The presuppositions of such therapy are to block the proliferation of cancer cells by interfering with specific molecules necessary for the successful tumour growth and development. Drugs are designed in such a way to affect a specific, targeted biochemical pathway [4]. The first and most crucial step in planning an effective treatment is to find specific molecules that serve as a molecular target. One of the methods is to identify proteins present in a tumour, but absent or exhibiting a decreased expression in normal, healthy cells. An- other approach is to look for changed proteins that drive cancer progression. These can be fusion proteins located in altered cells - the cause of genetic instability. Each cell has specific cell-surface receptors, which are responsible for activation of a response to extracellular stimuli. Often these proteins are used as the target of therapy because they show a relatively high frequency of mutation or overexpression [1-4].

\section{Molecularly targeted cancer therapy}

\section{Molecular targets}

An example of a protein that is a target of molecular therapy is Vascular Endothelial Growth Factor (VEGF), one of the most important factors regulating the development of tumour blood vessels, thereby modulating the process of angiogenesis. The angiogenesis process provides nutrients and oxygen for cancer cells, contributing to the formation and growth tumour. In addition, it allows the spread of cancer cells - VEGF stimulates the secretion of more proteases, contributing to the degradation of the basal membrane. [5] Inhibition of VEGF makes it possible to arrest the growth of blood 
vessels and cut off cancer cells from nutrients and oxygen [6]. There are two approaches used to disrupt VEGF signalling, i.e. including ligand blockade and pharmacological blocking Vascular Endothelial Growth Factor Receptor (VEGFR).

Another example of a molecular target is Epidermal Growth Factor Receptor (EGFR). EGFR is a transmembrane glycoprotein that is overexpressed on the surface of numerous tumour cells. Binding of the receptor to the ligand leads to the activation of a signalling cascade modulating proliferation, adhesion, migration, angiogenesis and metastasis. The use of monoclonal antibodies directed against EGFR causes binding and blocking of signalling pathways, which results in inhibition of tumour growth development and also in the prevention of metastases [6].

The next protein used as the molecular target is Human Epidermal Growth Factor Receptor 2 (HER2), is characterized by the external activity of tyrosine kinase. Gene amplification and overexpression of this protein is identified in approximately $20 \%$ of breast cancer cases, it is also a negative prognostic factor. HER2 is a key mediator of cell growth and differentiation. HER2-positive tumours show a higher degree of malignancy than other subtypes. Inhibition or binding of this receptor may prevent the activation of signalling pathways, thus also the proliferation of the changed tissue [7].

An example of gene serving as a target in personalized therapy is B-Raf Proto-Oncogene (BRAF). B-Raf protein is a kinase from the RAF family of proteins and participates in intercellular signalling. BRAF mutations as BRAFV600E and BRAFV600K occurs with a high frequency in various types of cancer, may be inherited or acquired during postnatal development. This kinase is part of the MAPK signalling pathway, which continuous activation leads to increased proliferation, higher invasiveness and survival of cancer cells, and also increases the probability of metastasis. Modern drugs inhibit the activity of the altered protein by inhibiting this kinase [8]

\section{Mechanism of action}

Two action mechanisms of a targeted anti-cancer treatment can be distinguished. The first of these is blocking proliferation through the use of small molecule tyrosine kinase inhibitors (TKI) which causes impeding the activation of the signalling pathway by blocking the action of an abnormal protein (Tab. 1) [1, 2].

The second type of treatment uses monoclonal antibodies that bind specifically to the target proteins, which leads to inhibition of their activity (Tab. 2). Through the use of genetic engineering, humanized and fully human monoclonal antibodies are produced and are used in the treatment of cancer [10].

\section{Toxicity of drugs used in targeted therapy}

\section{Inhibitors of the Epidermal Growth Factor Receptor (EGFR)}

Epidermal growth factor receptor inhibitors are approved for treatment of early-stage cancers, such as non-small cell lung cancer, colorectal cancer, breast cancer, pancreatic cancer, head and neck cancer and squamous cell carcinoma. They include intravenous monoclonal antibody treatment or oral therapy with small tyrosine kinase inhibitors. EGFR is expressed in the structures of the skin, so inhibition is associated with a number of adverse skin complications [11]. It is believed that this is related to the inhibition of EGFR in basal keratinocytes and hair follicles. They exhibit similar expression of EGFR as in cancer cells [12].

In clinical practice, one of the most commonly observed skin reactions is an acne-like eruption. It manifests itself with blister-like papules or pustules that occur in clusters. Aforementioned changes can cause itching, smarting, pain and irritation. In the first stage of treatment both, erythema and edema can be present. Papules may occur between the 2nd and 3rd week, and after about a month, persistent erythema, dryness and telangiectasia are commonly noted. In extreme cases, it is necessary to reduce the dose or completely terminate the treatment. Some patients notice an involution in post-treatment changes, but there are also cases in which severe and persistent acne-like reactions often develop [11, 13]. Research regarding this subject reports a correlation between the occurrence of a rash and the effectiveness of treatment. It has been confirmed that acne-like changes in the treatment with the use of gefitinib and erlotinib may be an effective clinical indicator for predicting responses in patients with small cell lung cancer [14].

Another common side effect of EGFR inhibitors is Hand-Foot Syndrome or Palmar-Plantar Erythrodysesthesia. In the case of sorafenib and sunitinib therapy, the intensity of these side effects is proportional to the dose [14]. Syndromes range from erythema, edema and burning, to hyperkeratosis. Those can significantly affect the well-being of the patient during therapy. Other skin complications include observable pathological changes in hair and nails, resulting in hair loss and brittleness, also nail brittleness, as well as discolouration or curling. [15]. Other side effects associated with EGFR inhibition include dry skin, pruritus, atrophy of the natural barrier protecting against infection, seborrheic dermatitis, subungual hemorrhage, photosensitivity or paronychia, manifested by purulent inflammation around the nails $[11,12]$.

Trastuzumab, a monoclonal antibody directed against HER2, is mainly applied in breast cancer. The most common side effects are chills, fever, asthenia and nausea. However, cases of extensive and toxic 
cardiovascular complications or even fatal pulmonary complications have also been reported. These severe events include primarily; dyspnea, pneumonia, lung infiltrates, edema, insufficiency or hypoxia. Usually, treatment termination can take place if initial symptoms indicating pulmonary complications occur $[16,17]$.

\section{Inhibitors of B-RAF serine-threonine kinase}

Skin lesions observed during treatment with EGFR inhibitors may also occur during targeted melanoma therapy. Increased risk of skin complications during vemurafenib or dabrafenib therapy has been observed in the range of 8 to 36 weeks after administration of the first dose [18]. During BRAF inhibitors monotherapy, skin toxicity is observed in $92-99 \%$ of patients, the most common being acne-like lesions, maculopapular or exudative rashes [19]. In addition, painful wart-like lesions most commonly occur after 4 weeks of treatment. Another frequent side effect is the Hand-Foot Syndrome described earlier. Most patients taking BRAF inhibitors suffer from hypersensitivity to ultraviolet $A$ radiation (UVA). Side effects associated with exposure to UVA are manifested by severe sunburns, pain or blisters. Other side effects that occur during treatment of melanoma include alopecia, seborrheic dermatitis, follicular keratosis, or epidermal cysts $[19,20]$. Treatment of skin lesions most often involves the use of antibiotics and corticosteroids, which carries additional toxicity to the patient. All described Dermatologic Events (DEAs) exert enormous influence on the mental, social and physical health of patients and affect their overall quality of life [18].
Other possibly dangerous complications, occurring during treatment with drugs such as dabrafenib or vemurafenib are fever, skin lesions, hepatic toxicity and lymphopenia. Lymphopenia makes it difficult to initiate an immune response and thus leads to numerous infections. The late identification of infections caused by pathogens can lead to particularly severe and potentially fatal diseases [21].

\section{Inhibitors of Vascular Endothelial Growth Factor (VEGF)}

In addition to skin complications, molecular targeted therapy can cause a number of other side effects. Tyrosine Kinase Inhibitors like sorafenib, sunitinib, imatinib ora axitinib may have side effects associated with hypothyroidism:

- thyroid atrophy by inhibiting its vascularization,

- preventing the binding of VEGF to normal thyroid cells,

- reducing the synthesis of thyroid hormones,

- inhibition of iodine uptake,

- sdrug-induced thyroid atrophy [22, 23].

Moreover, in 2010, thyroid dysfunction was described in patients treated with gefitinib [23]. These events were related to its mechanism of action, as it affects a lot of tyrosine kinases and binds VEGFR. The result of such interaction may be thyroid dysfunction occurring after less than a month of administrating the drug, it may have a place earlier than in the case of sorafenib or sunitinib [24].

VEGF plays a key role in the development of vascular blood vessels, which is why anti-angiogenic

Table 1. Examples of kinase inhibitors used in targeted therapy [8]

\begin{tabular}{llll}
\hline & Mechanism of action & Target & Therapeutic indications \\
\hline Dabrafenib & Selectively binds B-raf protein. & BRAF & melanoma \\
Vemurafenib & Selectively binds to the BRAP ATP binding site. & BRAF & melanoma \\
Lapatinib & $\begin{array}{l}\text { Reversibly blocks the phosphorylation of EGFR, ErbB2, } \\
\text { ERK-1 and-2 and AKT kinases. }\end{array}$ & EGFR & breast cancer \\
Erlotynib & $\begin{array}{l}\text { Reversibly binds to the intracellular catalytic domain of } \\
\text { the EGFR receptor. }\end{array}$ & EGFR & NSCLC, \\
& & & pancreatic cancer \\
\hline
\end{tabular}

Table 2. Examples of antibodies used in targeted therapy [8]

\begin{tabular}{|c|c|c|c|}
\hline & Mechanism of action & $\begin{array}{l}\text { Target and type of } \\
\text { monoclonal antibodies }\end{array}$ & Therapeutic indications \\
\hline Cetuximab & $\begin{array}{l}\text { Binds to the intracellular domain } \\
\text { of EGFR. }\end{array}$ & $\begin{array}{l}\text { EGFR } \\
\text { chimeric lgG1 }\end{array}$ & $\begin{array}{l}\text { colorectal cancer, } \\
\text { HNSCC }\end{array}$ \\
\hline Bavacizumab & $\begin{array}{l}\text { Inhibiting receptor activation } \\
\text { by binds to VEGF. }\end{array}$ & VEGF humanize IgG1 & $\begin{array}{l}\text { cervical cancer, NSCLC, } \\
\text { glioblastoma, ovarian cancer, kidney } \\
\text { cancer, colorectal cancer }\end{array}$ \\
\hline Trastuzumab & $\begin{array}{l}\text { binds HER2 on the surface } \\
\text { of tumor cells, bring cell-mediated } \\
\text { cytotoxicity in relation to tumor } \\
\text { cells that overexpress HER2. }\end{array}$ & HER2 humanize IgG1 & $\begin{array}{l}\text { Stomach or esophageal } \\
\text { adenocarcinoma, breast cancer }\end{array}$ \\
\hline
\end{tabular}


therapies focus on inhibiting this factor. Numerous inhibitors have been approved by the U.S. Food and Drug Administration (FDA) they show potential benefits but also cause significant dose-related complications. In 2011, the FDA withdrew the permission to use bevacizumab in case of treatment of breast cancer because the benefits did not outweigh the risks of the treatment $[25,26]$. Due to the mechanism of action, the majority of adverse reactions resulting from anti-VEGF therapy is associated with vascular disease. These include cardiac infarction, cerebral stroke, heart failure, hypertension, thromboembolism or proteinuria [25].

Hypertension is considered to be a frequent consequence of undergoing VEGF inhibitors therapy [25]. Induction of hypertension may be connected with inhibition of nitric oxide production in endothelial cells. It usually is asymptomatic, but it can be a risk factor for cardiovascular disease or renal failure [27]. Another common consequence of using anti-angiogenic drugs is proteinuria, most likely caused by the inhibition of VEGF paracrine signalling [25], or acute hypertension [12]. Proteinuria is associated with overproduction of abnormal proteins that can cause nephrotoxicity. The above side effects accompanying anti-VEGF therapy may correlate with each other or may be a result of long-term therapy. In the elderly, they are not a prognostic factor [25].

There is also a risk of arterial thromboembolic complications, as VEGF inhibition may negatively affect blood dust and von Willebrand factor, and consequently lead to the activation of the hemostasis system [12]. All agents directed against VEGF are associated with an increased risk of bleeding $[28,29]$.

\section{Anti-cancer drug resistance}

Drug resistance can be one of the most significant limiting factors during anti-cancer treatment. The resistance may result from the adaptation of tumour cells caused by regular drug application or from the presence of pre-existing changes at the molecular level $[30,2]$. Congenital cellular resistance is characterized by a lack of response to drugs from the beginning of their use. The acquired one, however, appears sometime after the start of therapy, most often after 12 or 18 months. Most patients develop resistance at one of the stages of treatment. There are many mutations and disorders that may result in the cell not being sensitive to targeted treatment [2, 31]. Asic K., in his work from 2016, divided the resistance mechanisms into:

- disturbances of drug penetration inside the cell - changes occurring in genes coding for target proteins,

- addiction to the alternative signalling pathway - activation of a protein that performs a similar function, causing further growth of cancer cells,
- changes leading to further activation of the target path - mutations or changes of genes encoding proteins below or above the target molecule,

- replacement/imitation of the target function,

- activation at Multidrug Resistance (MDR) [31].

\section{Inhibitors of the Epidermal Growth Factor}

Receptor (EGFR)

In the case of treatment of non-small cell lung cancer (NSCLC) with erlotinib, all previously mentioned mechanisms have been observed, both congenital and acquired cellular resistance. During the treatment of colorectal cancer with cetuximab, in the case of congenital resistance, compensation of the EGFR function and changes in the regulation of the target signal pathway have been demonstrated. This resulted in the further activity of the EGFR molecule. In the example of acquired resistance, changes indicating contact with the drug have been shown [31, 32]. The primary problem in NSCLC therapy is the activation of alternative, parallel signalling pathways that cause the blockade created by the drug to be bypassed. One of the additional mechanisms of potential resistance is the activation of the insulin-like receptor 1 (IGFR1) [33].

Similarly, targeted therapy for HER2 overexpression in the treatment of breast cancer using monoclonal antibodies may contribute to all of the previously mentioned mechanisms of cellular resistance. In the case of acquired resistance, MDR was excluded as well as replacement/imitation of the target function. When kinase inhibitors are used, further activation of the target signalling pathway and replacement/imitation of the target functions by activation of another protein or MDR may also occur [31]. Trastuzumab is an example of a drug that allows considerable clinical advances in the treatment of breast cancer. The impediments, however, is not fully understood. Changes in the cell cycle, DNA repair mechanisms, PI3K signalling pathway, as well as inhibition of the extracellular protein domain or HER2 degradation are indicated. In the case of trastuzumab, the mechanism of impaired binding of the drug to the receptor may refer to the formation of a truncated HER2 protein, devoid of the extracellular domain. Receptor cloning by MUC4 glycoprotein has also been described. The loss of the PTEN suppressor gene (Phosphatase and Tensin Homolog), or excessive activation of the PI3K-AKT pathway, results in the change of the signalling pathway into its alternative counterpart [33].

\section{Inhibitors of B-RAF serine-threonine kinase}

Mechanisms of congenital resistance occurring during treatment of melanoma with the BRAFV600E mutation using vemurafenib, include dependence on 
alternative signalling pathways, further activation of the target pathway and imitation of the target function. In acquired resistance, it is also possible to make difficult contact with the drug by incomplete binding to the cancer cell. The use of sunitinib is associated with the occurrence of all the previously mentioned mechanisms of congenital cellular resistance, with the exception of the blockade of contact with the drug. Likewise, further activation of the target path was excluded from the acquired resistance [31]. In addition, it has been shown that by reducing tumour vasculature when using sunitinib, cancer cells acquiring resistance more often form distant metastases [34].

Under physiological conditions, ERK signaling is regulated by feedback mechanisms to preserve homeostasis of the body and normal cell growth. In tumors with the KRAS or BRAF mutation, this signaling disorders are often noticed and the phenomenon of overexpression of the path prompted researchers to use inhibitors of this ERK signaling as a therapeutic agent - dabrafenib and vemurafenib. Treatment has shown that effectiveness correlates with the braking force of signaling. This is related to the rapid adaptation of the tumor to the inhibited pathway through mechanisms leading to RAF dimerization and the increased level of ERK signaling. Vemurafenib and dabrafenib require a monomeric RAF protein for inhibition. Therefore, when it is dimerized, the action of drugs is inhibited. Furthermore, tumor heterogeneity influence to the development of resistance to individual factors [35].

\section{The cost of targeted therapies}

The main premise of targeted therapies requires the use of state-of-the-art diagnostic technologies, blood and tissue banks, and the use of extended clinical knowledge, which significantly influences the cost of the final treatment. Drugs used in such therapies are designed to work on a specified biochemical pathway and require extensive trials, often limited to selected patient populations. Therefore, except for the biological barriers, which are often related to the mechanisms of resistance, also incomplete understanding of signaling pathways and further search for useful biomarkers, the widespread use of personalized medicine is limited by the economic barrier. There are various reasons why investors have doubts about the profitability of personalized treatment. One of the main sources of hesitation is the problem of identification of appropriate diagnostic technologies that would be both, effective and at the same time inexpensive [36].

Expensive studies can be profitable only if, due to their high accuracy, they bring significant health benefits to well-identified target groups. Standard breast cancer treatment is based on chemotherapy and hormonotherapy. In targeted treatment, other than the standard characteristics of a tumor, such as size, lymph node metastases, or hormone levels, tests are performed in order to detect the expression of tumor genes and protein levels. An example is the HER2 receptor study to select candidates for trastuzumab treatment. It is recommended to apply either routine immunohistochemistry (IHC) or Fluorescent in situ Hybridization (FISH). However, they differ in accuracy and efficiency, so researchers often have to do carry out both tests. Only patients with a HER2-positive result are classified for this therapy [37].

A relatively expensive study in targeted therapy at HER2 is a Histopathological analysis which uses antibodies or Gene Expression Profiling (GEP); as a tool for risk stratification. GEP includes gene analysis that uses either DNA microarray technology or polymerase chain reaction in real time (Reverse Transcriptase PCR, RT-PCR) [37, 38]. Total cost of therapy for trastuzumeb of early-stage HER-positive breast cancer averages around \$67,800 [39]. Treatment with trantuzumab with HER-positive stomach cancer is estimated at $\$ 90,000$ a year [40].

FDA-approved Vemurafenib, used in the treatment of metastatic melanoma in patients with the BRAF mutation, is implemented instead of the standard dacarbazine treatment. Curl et al. compared the cost-effectiveness of both treatments. It was shown that the cost of using vemurafenib is inadequate to its results [41].

Comparative analysis indicates that the overall cost of targeted therapy may outweigh the benefits of the treatment. In 2011, the majority of targeted drugs approved by the FDA, assuming a 12-month treatment, required a cost of $\$ 20,000$, which resulted in insurance companies starting to withhold their expenses for treatment. The costs of targeted therapy fell completely on the patient, which in many cases led to stressful situations resulting from the loss of financial liquidity an example may be bevacizumab approved for treatment, among others colorectal cancer, NSCLC or ovarian cancer. The annual cost of the treatment is estimated around $\$ 50,000$, which in some cases can amount to $\$ 100,000$. Finally, the monoclonal antibody was withdrawn from the treatment of breast cancer due to proven ineffectiveness compared to standard therapy, while the cost of treating the patient for one year could be as high as $\$ 500,000$. Another drug, the use of which requires large financial outlays is cetuximab, approved for the treatment of among others NSCLC. Patient's therapy with its use costs about $\$ 800,000$ a year. Even if there are funds for treatment, this does not ensure survival, but it may have many side effects that adversely affect the patient's well-being [40]. Another problem that consumes large financial outlays is the costs of mitigation or treatment of side effects. In contrast to diagnostics, they are not included in the estimated cost of therapy [41]. 


\section{Conclusions}

Molecular Targeted Therapy is one of the modern tools used in the fight against such cancers as melanoma, breast cancer or colorectal cancer. The condition for its use is accurate diagnostics which enables the characterization of cancer cells. The most important element of this therapy is to find a molecular target. Novel, highly reliable markers for targeting cancer cells with a specific phenotype are still being sought.

In most patients, drug resistance appears after a certain time, despite the initial effectiveness of the implemented therapy. After the acquisition of resistance, it seems almost impossible to overcome the mechanisms allowing cancer cells to further undisturbed proliferation. Most often in this case the treatment with personalized therapy is completed and the patient is treated with standard methods. There have also been attempts to circumvent resistance mechanisms through the use of combination therapies, but this is always associated with increased costs of treatment.

Another problem in the use of personalized therapy is high toxicity. Although the effect of therapy is targeted, it still affects normal cells. Some cells in the body show expression of molecules that serve as a molecular target, but not as pathological as in cancer cells. For this reason, drugs directed against EGFR or HER2 most often cause dangerous skin changes, anti-angiogenic drugs, and cardiovascular complications. The increase of side effects is associated with the reduction in the dose of the drug or, in extreme cases, termination of therapy. Mechanisms of resistance and toxicity of therapy chiefly reduce its effectiveness.

The additional factor is the costs of diagnostics, medicines and combating side effects. They predominantly contribute to the unprofitability of treatment. If the effects are inadequate to the incurred costs and they additionally reduce the patient's quality of life, the drugs are not approved, and even though they were previously registered, they are removed from circulation.

The limitations of molecular targeted therapy are in many cases greater than with standard anti-cancer therapies. Meeting the requirements for reducing side effects and costs with simultaneously higher efficacy would require using molecules that are found on cancer cells as a molecular target, but are absent on normal cells (Fig. 1). Expanding our knowledge of the resistance mechanisms and signaling pathways in tumors would increase the chance of success for targeted therapy. It is equally important to look for new methods of drug production and diagnostic tests that would minimize the cost of treatment.

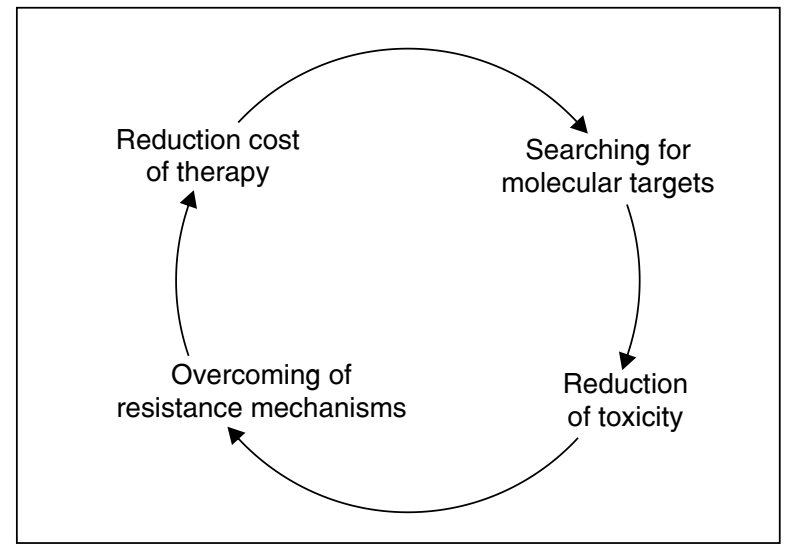

Figure 1. Perspectives for Molecularly targeted therapy

\section{Disclosure of interest}

The authors declare no conflict of interest.

\section{List of abbreviations}

BRAF - B-Raf Proto-Oncogene

DEAs - Dermatologic Events

EGFR - Epidermal Growth Factor Receptor

FDA - Food and Drug Administration

FISH — Fluorescent In Situ Hybridization

GEP - Gene Expression Profiling

HER2 - Human Epidermal Growth Factor Receptor 2

HNSCC - Head and neck squamous cell carcinoma

IGFR1 — Insulin-Like Growth Factor 1

IHC - Immunohistochemistry

MDR - Multidrug Resistance

NSCLC - Non-Small-Cell Lung Carcinoma

PMPY - Per Member Per Year

PTEN - Phosphatase And Tensin Homolog deleted on chromosome ten

RT-PCR - Reverse Transcriptase PCR

TKI - Tyrosine Kinase Inhibitor

UVA - Ultraviolet A

VEGFRs - Vascular Endothelial Growth Factor Receptor

VEGR — Vascular Endothelial Growth Factor

\section{References}

1. Huang M, Shen A, Ding J, et al. Molecularly targeted cancer therapy: some lessons from the past decade. Trends Pharmacol Sci. 2014; 35(1): 41-50, doi: 10.1016/j.tips.2013.11.004, indexed in Pubmed: 24361003

2. Krajewski KM, Braschi-Amirfarzan M, DiPiro PJ, et al. Molecular Targeted Therapy in Modern Oncology: Imaging Assessment of Treatment Response and Toxicities. Korean J Radiol. 2017; 18(1): 28-41, doi: 10.3348/kjr.2017.18.1.28, indexed in Pubmed: 28096716. 
3. Meric-Bernstam F, Mills GB. Overcoming implementation challenges of personalized cancer therapy. Nat Rev Clin Oncol. 2012; 9(9): 542-548, doi: 10.1038/nrclinonc.2012.127, indexed in Pubmed: 22850751

4. Targeted Cancer Therapies. National Cancer Institute. https://www cancer.gov/about-cancer/treatment/types/targeted-therapies/targeted-therapies-fact-sheet (22.01.2019)

5. Zuazo-Gaztelu I, Casanovas O. Unraveling the Role of Angiogenesis in Cancer Ecosystems. Front Oncol. 2018; 8: 248, doi: 10.3389/fonc.2018.00248, indexed in Pubmed: 30013950.

6. Ohhara Y, Fukuda N, Takeuchi S, et al. Role of targeted therapy in metastatic colorectal cancer. World J Gastrointest Oncol. 2016; 8(9): 642-655, doi: 10.4251/wjgo.v8.i9.642, indexed in Pubmed: 27672422

7. Jackisch $C$, Lammers $P$, Jacobs I. Evolving landscape of human epidermal growth factor receptor 2-positive breast cancer treatment and the future of biosimilars. Breast. 2017; 32: 199-216, doi: 10.1016/j. breast.2017.01.010, indexed in Pubmed: 28236776

8. Vennepureddy A, Thumallapally N, Motilal Nehru V, et al. Novel Drugs and Combination Therapies for the Treatment of Metastatic Melanoma. J Clin Med Res. 2016; 8(2): 63-75, doi: 10.14740/jocmr2424w, indexed in Pubmed: 26767073.

9. $\mathrm{NCl}$ Drug Dictionary. National Cancer Institute. https://www.cancer gov/publications/dictionaries/cancer-drug (23.01.2019).

10. Powroźnik B, Kubowicz P, Pękala E. Monoclonal antibodies in targeted therapy. Postepy Hig Med Dosw (Online). 2012; 66: 663-673, doi: 10.5604/17322693.1009980, indexed in Pubmed: 23001208

11. Lupu I, Voiculescu N, Bacalbasa N, et al. Cutaneous complications of molecular targeted therapy used in oncology. J Med Life. 2016; 9(1): 19-25, indexed in Pubmed: 27974909.

12. Holcmann M, Sibilia M. Mechanisms underlying skin disorders induced by EGFR inhibitors. Mol Cell Oncol. 2015; 2(4): e1004969, doi: 10.10 80/23723556.2015.1004969, indexed in Pubmed: 27308503

13. Scope A, Agero AL, Dusza SW, et al. Randomized double-blind trial of prophylactic oral minocycline and topical tazarotene for cetuximab-associated acne-like eruption. J Clin Oncol. 2007; 25(34): 5390-5396, doi: 10.1200/JCO.2007.12.6987, indexed in Pubmed: 18048820

14. Liu Hb, Wu Y, Lv Tf, et al. Skin rash could predict the response to EGFR tyrosine kinase inhibitor and the prognosis for patients with non-small cell lung cancer: a systematic review and meta-analysis. PLoS One. 2013; 8(1) e55128, doi: 10.1371/journal.pone.0055128, indexed in Pubmed: 23383079

15. Rinderknecht JD, Goldinger SM, Rozati S, et al. RASopathic skin eruptions during vemurafenib therapy. PLoS One. 2013; 8(3): e58721, doi: 10.1371/journal.pone.0058721, indexed in Pubmed: 23516541.

16. Adjuvant Breast Cancer Treatment Side Effects. Herceptin (trastuzumab). http://www.herceptin.com/hcp/treatment/adjuvant/side-effects, (24.01.2019.)

17. Vogel CL, Cobleigh MA, Tripathy D, et al. Efficacy and safety of trastuzumab as a single agent in first-line treatment of HER2-overexpressing metastatic breast cancer. J Clin Oncol. 2002; 20(3): 719-726, doi: 10.1200/JCO.2002.20.3.719, indexed in Pubmed: 11821453.

18. Tang N, Ratner D. Managing Cutaneous Side Effects From Targeted Molecular Inhibitors for Melanoma and Nonmelanoma Skin Cancer. Dermatol Surg. 2016; 42 Suppl 1: S40-S48, doi: 10.1097/DSS.0000000000000519, indexed in Pubmed: 26730973.

19. de Golian E, Kwong BY, Swetter SM, et al. Cutaneous Complications of Targeted Melanoma Therapy. Curr Treat Options Oncol. 2016; 17(11) 57, doi: 10.1007/s11864-016-0434-0, indexed in Pubmed: 27645330.

20. Welsh SJ, Corrie PG. Management of BRAF and MEK inhibitor toxicities in patients with metastatic melanoma. Ther Adv Med Oncol. 2015; 7(2): 122-136, doi: 10.1177/1758834014566428, indexed in Pubmed: 25755684

21. Sondermann W, Griewank KG, Schilling B, et al. Corticosteroids augment BRAF inhibitor vemurafenib induced lymphopenia and risk of infection. PLoS One. 2015; 10(4): e0124590, doi: 10.1371/journal. pone.0124590, indexed in Pubmed: 25897843

22. Shu M, Zai X, Zhang B, et al. Hypothyroidism Side Effect in Patients Treated with Sunitinib or Sorafenib: Clinical and Structural Analyses. PLoS One. 2016; 11(1): e0147048, doi: 10.1371/journal. pone.0147048, indexed in Pubmed: 26784451
23. Mukohara T, Nakajima H, Mukai H, et al. Effect of axitinib (AG-013736) on fatigue, thyroid-stimulating hormone, and biomarkers: a phase I study in Japanese patients. Cancer Sci. 2010; 101(4): 963-968 doi: 10.1111/j.1349-7006.2009.01465 x indexed in Pubmed: 20180805.

24. Daimon $M$, Kato $T$, Kaino $W$, et al. Thyroid dysfunction in patients treated with tyrosine kinase inhibitors, sunitinib, sorafenib and axitinib for metastatic renal cell carcinoma. Jpn J Clin Oncol. 2012; 42(8): 742-747, doi: 10.1093/jico/hys076, indexed in Pubmed: 22628612.

25. Faruque LI, Lin M, Battistella M, et al. Systematic review of the risk of adverse outcomes associated with vascular endothelial growth factor inhibitors for the treatment of cancer. PLoS One. 2014; 9(7): e101145, doi: 10.1371/journal.pone.0101145, indexed in Pubmed: 24988441.

26. Sasich LD, Sukkari SR. The US FDAs withdrawal of the breast cancer indication for Avastin (bevacizumab). Saudi Pharm J. 2012; 20(4): 381385, doi: 10.1016/j.jsps.2011.12.001, indexed in Pubmed: 23960813.

27. Feliu J, Salud A, Safont MJ, et al. Correlation of hypertension and proteinuria with outcome in elderly bevacizumab-treated patients with metastatic colorectal cancer. PLoS One. 2015; 10(1): e0116527, doi: 10.1371/journal.pone.0116527, indexed in Pubmed: 25602286.

28. Zuo PY, Chen XL, Liu YW, et al. Increased risk of cerebrovascular events in patients with cancer treated with bevacizumab: a meta-analysis. PLoS One. 2014; 9(7): e102484, doi: 10.1371/journal. pone.0102484, indexed in Pubmed: 25025282.

29. Guan M, Zhou YP Sun لJ et al. Adverse events of monoclonal antibodies used for cancer therapy. Biomed Res Int. 2015; 2015: 428169, doi: 10.1155/2015/428169, indexed in Pubmed: 26075239

30. Styczyński J, Haus O. [Cytogenetics and in vitro drug resistance of acute leukemia in children and adults]. Postepy Hig Med Dosw (Online). 2006; 60: 527-537, indexed in Pubmed: 17060894

31. Asić K. Dominant mechanisms of primary resistance differ from dominant mechanisms of secondary resistance to targeted therapies. Crit Rev Oncol Hematol. 2016; 97: 178-196, doi: 10.1016/j. critrevonc.2015.08.004, indexed in Pubmed: 26364890.

32. Spaans JN, Goss GD. Drug resistance to molecular targeted therapy and its consequences for treatment decisions in non-small-cell lung cancer. Front Oncol. 2014; 4: 190, doi: 10.3389/fonc.2014.00190, indexed in Pubmed: 25101246.

33. Fiszman GL, Jasnis MA. Molecular Mechanisms of Trastuzumab Resistance in HER2 Overexpressing Breast Cancer. Int J Breast Cancer. 2011; 2011: 352182, doi: 10.4061/2011/352182, indexed in Pubmed: 22295219

34. Wragg JW, Heath VL, Bicknell R. Sunitinib Treatment Enhances Metastasis of Innately Drug-Resistant Breast Tumors. Cancer Res. 2017; 77(4): 1008-1020, doi: 10.1158/0008-5472.CAN-16-1982, indexed in Pubmed: 28011623

35. Samatar AA, Poulikakos PI. Targeting RAS-ERK signalling in cancer: promises and challenges. Nat Rev Drug Discov. 2014; 13(12): 928-942, doi: 10.1038/nrd4281, indexed in Pubmed: 25435214.

36. Jakka S, Rossbach M. An economic perspective on personalized medicine. The HUGO Journal. 2013; 7(1): 1, doi: 10.1186/1877-6566-7-1.

37. Elkin EB, Marshall DA, Kulin NA, et al. Economic evaluation of targeted cancer interventions: critical review and recommendations. Genet Med. 2011; 13(10): 853-860, doi: 10.1097/GIM.0b013e31821f3e64, indexed in Pubmed: 21637102

38. RaabSS. The cost-effectiveness of immunohistochemistry. ArchPathol LabMed. 2000; 124(8): 1185-1191, doi: 10.1043/0003-9985(2000)124<1185:TCEOI>2.0. CO;2, indexed in Pubmed: 10923081

39. Leung W, Kvizhinadze G, Nair N, et al. Adjuvant Trastuzumab in HER2-Positive Early Breast Cancer by Age and Hormone Receptor Status: A Cost-Utility Analysis. PLoS Med. 2016; 13(8): e1002067, doi: 10.1371/journal.pmed. 1002067, indexed in Pubmed: 27504960

40. Jackson DB, Sood AK. Personalized cancer medicine--advances and socio-economic challenges. Nat Rev Clin Oncol. 2011; 8(12): 735-741, doi: 10.1038/nrclinonc.2011.151, indexed in Pubmed: 21989071.

41. Curl P, Vujic I, van 't Veer LJ, et al. Cost-effectiveness of treatment strategies for BRAF-mutated metastatic melanoma. PLoS One. 2014; 9(9): e107255, doi: 10.1371/journal.pone.0107255, indexed in Pubmed: 25198196. 